CTEQ/408

MSUHEP-50319

April, 1995

\title{
Effects of QCD Resummation on Distributions of Leptons from the Decay of Electroweak Vector Bosons
}

\author{
Csaba Balázs ${ }^{(a)}$, Jianwei Qiu ${ }^{(b)}$ and C.-P. Yuan ${ }^{(a)}$ \\ (a) Department of Physics and Astronomy, Michigan State University \\ East Lansing, MI 48824, U.S.A. \\ (b) Department of Physics and Astronomy, Iowa State University \\ Ames, IA 50011, U.S.A.
}

\begin{abstract}
We study the distributions of leptons from the decay of electroweak vector bosons produced in hadron collisions. The effects of the initial state multiple soft-gluon emission, using the Collins-Soper resummation formalism, are included. The resummed results are compared with the next-to-leading-order results for the distributions of the transverse momentum, rapidity asymmetry, and azimuthal angle of the decay leptons.
\end{abstract}




\section{Introduction}

With the discovery of the top quark [1], the electroweak symmetry breaking mechanism remains one of the major mysteries of particle physics today. Unfortunately, the precision low energy data have told us very little about the scalar sector, i.e. the electroweak symmetry breaking sector, of the Standard Model (SM). From CERN LEP data we learned that the mass $m_{H}$ of the Higgs boson has to be larger than about $60 \mathrm{GeV}$ [2]. However, the precision low energy data do not exclude the possibility for $m_{H}$ to be in the order of $1 \mathrm{TeV}$. At the moment, one of the largest theoretical errors in analyzing radiative corrections to low energy data comes from the prediction of the fine structure constant $\alpha$ evaluated at the $Z$-boson mass scale, due to the less precise low energy $e^{-} e^{+} \rightarrow$ hadrons data [3]. Without further improvement in the determination of $\alpha\left(M_{Z}\right)$, a more precise way to test the SM is to have a better measurement of $M_{W}$. If $M_{W}$ is measured within $40 \mathrm{MeV}$ and the mass of the top quark within $4 \mathrm{GeV}$, then $m_{H}$ can be constrained within a couple of hundred $\mathrm{GeV}$ [4]. To reach such an accuracy in the measurement of $M_{W}$ at hadron colliders, we have to know the kinematics of the $W^{ \pm}$-boson well. Since $W^{+}$decays into a charged lepton $\ell^{+}$and a neutrino $\nu_{\ell}$, the kinematics of the $W^{+}$cannot be accurately known because of the missing momentum carried by $\nu_{\ell}$. It is therefore desirable to have a good prediction on the kinematics of $\ell^{ \pm}$from the decay of $W^{ \pm}$.

The well established fact that the transverse momentum $Q_{T}$ distribution of the $W^{ \pm}$-boson cannot be described by the next-to-leading-order (NLO) perturbative calculation in low $Q_{T}$ region [5] implies that the transverse momentum $p_{T}^{\ell}$ of the lepton $\ell^{ \pm}$cannot be accurately predicted by the NLO calculation, especially for $p_{T}^{\ell} \sim M_{W} / 2$ (mostly with low $Q_{T}$ ) where the data dominate. We must resum the effects of the initial state multiple soft-gluon emission to predict the distributions of the leptons from the decay of the vector boson $V\left(=\gamma, W^{ \pm}\right.$or $\left.Z\right)$ produced in hadron collisions. In this paper, we adopt the Collins-Soper formalism [6], and closely follow the notation

\footnotetext{
${ }^{1}$ The analytic results presented in the paper also apply to the non-standard weak gauge boson(s) (such as $Z^{\prime}$ ) present in any extended gauge theory.
} 
used in Ref. [7] to resum the multiple soft-gluon effects to the transverse momentum, rapidity asymmetry, and azimuthal angle distributions of the decay product leptons.

\section{The Resummation Formalism}

To obtain the resummed results, we use dimensional regularization to regulate the infrared (IR) divergencies, and adopt the canonical- $\gamma_{5}$ prescription to calculate the anti-symmetric part of the matrix element in $n$-dimensional space-time. I $^{2}$ The infrared-anomalous contribution arising from using the canonical- $\gamma_{5}$ prescription was carefully handled by applying the procedures outlined in Ref. 10 for calculating both the virtual and the real diagrams. [

The kinematics of the vector boson $V$ (real or virtual) can be expressed in the terms of its mass $Q$, rapidity $y$, transverse momentum $Q_{T}$, and azimuthal angle $\phi_{V}$, measured in the laboratory frame. The kinematics of the leptons from the decay of the vector boson can be described by the polar angle $\theta$ and the azimuthal angle $\phi$, defined in the Collins-Soper frame [11], which is a special rest frame of the $V$-boson [12. The four-momentum of the decay product fermion in the lab frame isf

$$
L^{\mu}=\frac{Q}{2}\left(\frac{q^{\mu}}{Q}+\sin \theta \cos \phi X^{\mu}+\sin \theta \sin \phi Y^{\mu}+\cos \theta Z^{\mu}\right),
$$

where

$$
\begin{aligned}
q^{\mu} & =\left(M_{T} \cosh y, Q_{T} \cos \phi, Q_{T} \sin \phi, M_{T} \sinh y\right), \\
X^{\mu} & =-\frac{Q}{Q_{T} M_{T}}\left(q_{+} n^{\mu}+q_{-} \bar{n}^{\mu}-\frac{M_{T}^{2}}{Q^{2}} q^{\mu}\right) \\
Z^{\mu} & =\frac{1}{M_{T}}\left(q_{+} n^{\mu}-q_{-} \bar{n}^{\mu}\right), \\
Y^{\mu} & =\varepsilon^{\mu \nu \alpha \beta} \frac{q_{\nu}}{Q} Z_{\alpha} X_{\beta} .
\end{aligned}
$$

Here, $q_{ \pm}=\frac{1}{\sqrt{2}}\left(q^{0} \pm q^{3}\right), M_{T}=\sqrt{Q^{2}+Q_{T}^{2}}, n^{\nu}=\frac{1}{\sqrt{2}}(1,0,0,1)$ and $\bar{n}^{\nu}=\frac{1}{\sqrt{2}}(1,0,0,-1)$.

\footnotetext{
${ }^{2}$ In this prescription, $\gamma_{5}$ anticommutes with other $\gamma^{\prime}$ 's in the first four dimensions and commutes in others [8, 9].

${ }^{3}$ In Ref. [10] the authors calculated the anti-symmetric structure function $F_{3}$ for deep-inelastic scattering.

${ }^{4}$ Our convention is that $q^{\mu}=\left(q^{0}, q^{1}, q^{2}, q^{3}\right), Q=\sqrt{q^{2}}$, and $Q_{T}=\sqrt{\left(q^{1}\right)^{2}+\left(q^{2}\right)^{2}}$. The total anti-symmetric tensor $\epsilon^{0123}=-1$. The proton beam direction is assigned to be the positive z-axis.
} 
To obtain the fully differential cross section of the vector boson production and decay for all values of $Q_{T}$, we need the resummation formula [7]:

$$
\begin{aligned}
& \left(\frac{d \sigma\left(A B \rightarrow V\left(\rightarrow l \bar{l}^{\prime}\right) X\right)}{d Q^{2} d y d Q_{T}^{2} d \phi_{V} d \cos \theta d \phi}\right)_{r e s}=\frac{1}{96 \pi^{2} S} \frac{Q^{2}}{\left(Q^{2}-M_{V}^{2}\right)^{2}+M_{V}^{2} \Gamma_{V}^{2}} \\
& \quad \times\left\{\frac{1}{(2 \pi)^{2}} \int d^{2} b e^{i \vec{q}_{T} \cdot \vec{b}} \sum_{j, k} \widetilde{W}_{j k}\left(b_{*}, Q, x_{A}, x_{B}, \theta, \phi\right) F_{j k}^{N P}\left(b, Q, x_{A}, x_{B}\right)\right. \\
& \left.\quad+Y\left(Q_{T}, Q, x_{A}, x_{B}, \theta, \phi\right)\right\}
\end{aligned}
$$

Here $\widetilde{W}_{j k}$ is

$$
\begin{aligned}
& \widetilde{W}_{j k}\left(b, Q, x_{A}, x_{B}, \theta, \phi\right)=\exp \{-S(b, Q)\}\left|V_{j k}\right|^{2} \\
& \times\left\{\left[\left(C_{j a} \otimes f_{a / A}\right)\left(x_{A}\right)\left(C_{\bar{k} b} \otimes f_{b / B}\right)\left(x_{B}\right)+\left(C_{\bar{k} a} \otimes f_{a / A}\right)\left(x_{A}\right)\left(C_{j b} \otimes f_{b / B}\right)\left(x_{B}\right)\right]\right. \\
& \quad \times\left(g_{L}^{2}+g_{R}^{2}\right)\left(f_{L}^{2}+f_{R}^{2}\right)\left(1+\cos ^{2} \theta\right) \\
& +\left[\left(C_{j a} \otimes f_{a / A}\right)\left(x_{A}\right)\left(C_{\bar{k} b} \otimes f_{b / B}\right)\left(x_{B}\right)-\left(C_{\bar{k} a} \otimes f_{a / A}\right)\left(x_{A}\right)\left(C_{j b} \otimes f_{b / B}\right)\left(x_{B}\right)\right] \\
& \left.\quad \times\left(g_{L}^{2}-g_{R}^{2}\right)\left(f_{L}^{2}-f_{R}^{2}\right)(2 \cos \theta)\right\},
\end{aligned}
$$

where $\otimes$ denotes the convolution and is defined by

$$
\left(C_{j a} \otimes f_{a / A}\right)\left(x_{A}\right)=\int_{x_{A}}^{1} \frac{d \xi_{A}}{\xi_{A}} f_{a / A}\left(\xi_{A}, \mu\right) C_{j a}\left(\frac{x_{A}}{\xi_{A}}, b, \mu\right)
$$

and the $V_{j k}$ coefficients are given by

$$
V_{j k}= \begin{cases}\text { Cabibbo }- \text { Kobayashi }- \text { Maskawa matrix elements } & \text { for } V=W^{ \pm} \\ \delta_{j k} & \text { for } V=Z^{0}, \gamma\end{cases}
$$

In the above expressions $j$ represents quark flavors and $\bar{k}$ stands for anti-quark flavors. The dummy indices $a$ and $b$ are meant to sum over quarks and anti-quarks or gluons. Summation on these double indices is implied.

The Sudakov form factor $S(b, Q)$ is defined as

$$
S(b, Q)=\int_{b_{0}^{2} / b^{2}}^{Q^{2}} \frac{d \bar{\mu}^{2}}{\bar{\mu}^{2}}\left[\ln \left(\frac{Q^{2}}{\bar{\mu}^{2}}\right) A\left(\alpha_{s}(\bar{\mu})\right)+B\left(\alpha_{s}(\bar{\mu})\right)\right] .
$$

The $A, B$ functions and the Wilson coefficients $C_{j a}$, etc., were given in Ref. [0]. After fixing the renormalization constants $C_{1} \equiv b_{0}=2 e^{-\gamma_{E}}$ and $C_{2}=1$, one can obtain 
$A^{(1)}, B^{(1)}, A^{(2)}$ and $B^{(2)}$ from the Eqs. (3.19) to (3.22) of Ref. [7]. constant.) After choosing $\mu$ such that $\mu b \equiv C_{3}=2 e^{-\gamma_{E}}$, the Wilson coefficients $C_{j a}^{(i)}$ for the parity-conserving part of the resummed result are greatly simplified from the Eqs. (3.23) to (3.26) of Ref. [7] as

$$
C_{j k}^{(1)}=\delta_{j k}\left\{\frac{2}{3}(1-z)+\frac{1}{3}\left(\pi^{2}-8\right) \delta(1-z)\right\} \quad \text { and } \quad C_{j g}^{(1)}=\frac{1}{2} z(1-z) .
$$

Following the procedures given in Ref. [10] for handling the $\gamma_{5}$ 's in $n$-dimensional space-time, we find that the same Wilson coefficients $C_{j a}^{(i)}$ also apply to the parityviolating part of the resummed result.

In Eq. (3), the impact parameter $b$ is to be integrated from 0 to $\infty$. However, for $b \geq b_{\max }$, which corresponds to an energy scale less than $1 / b_{\max }$, the QCD coupling $\alpha_{s}$ becomes so large that a perturbative calculation is no longer reliable.甲 Hence, the non-perturbative function $F^{N P}$ is needed in the formalism, and generally has the structure

$$
F_{j k}^{N P}\left(b, Q, Q_{0}, x_{A}, x_{B}\right)=\exp \left[-\ln \left(\frac{Q^{2}}{Q_{0}^{2}}\right) h_{1}(b)-h_{j / A}\left(x_{A}, b\right)-h_{\bar{k} / B}\left(x_{B}, b\right)\right],
$$

where $h_{1}, h_{j / A}$ and $h_{\bar{k} / B}$ cannot be calculated using perturbation theory, so they must be measured experimentally. Furthermore, $\widetilde{W}$ is evaluated at $b_{*}$, with

$$
b_{*}=\frac{b}{\sqrt{1+\left(b / b_{\max }\right)^{2}}}
$$

such that $b_{*}$ never exceeds $b_{\max }$.

The $Y$-term in Eq. (3) is defined as

$$
\begin{aligned}
& Y\left(Q_{T}, Q, x_{A}, x_{B}, \theta, \phi\right)=\int_{x_{A}}^{1} \frac{d \xi_{A}}{\xi_{A}} \int_{x_{B}}^{1} \frac{d \xi_{B}}{\xi_{B}} \sum_{N=1}^{\infty}\left[\frac{\alpha_{s}(Q)}{\pi}\right]^{N} \\
& \quad \times f_{a / A}\left(\xi_{A} ; Q\right) R_{a b}^{(N)}\left(Q_{T}, Q, z_{A}, z_{B}, \theta, \phi, Q\right) f_{b / B}\left(\xi_{B} ; Q\right),
\end{aligned}
$$

in which the functions $R_{a b}^{(N)}$ only contain contributions which are less singular than $Q_{T}^{-2} \times(\operatorname{logs}$ or 1$)$ as $Q_{T} \rightarrow 0$. Their explicit expressions for $\overline{\mathrm{p} p} \rightarrow V\left(\rightarrow l \bar{l}^{\prime}\right) X$ are given in the Appendix.

\footnotetext{
${ }^{5}$ For instance, we obtain $A^{(1)}=4 / 3$ and $B^{(1)}=-2$. In our numerical results we also include $A^{(2)}$ and $B^{(2)}$.

${ }^{6}$ We use $b_{\max }=0.5 \mathrm{GeV}^{-1}$ in our calculation.
} 


\section{$3 \quad$ Numerical Results}

In this paper, we only give our numerical results for $\overline{\mathrm{p}} \mathrm{p} \rightarrow W^{+}\left(\rightarrow \ell^{+} \nu_{\ell}\right) X$ at the Fermilab Tevatron with $\sqrt{S}=1.8 \mathrm{TeV}$. The CTEQ3M parton distribution functions (PDF's) are used along with the non-perturbative function [13]

$$
F^{N P}\left(b, Q, Q_{0}, x_{A}, x_{B}\right)=\exp \left\{-g_{1} b^{2}-g_{2} b^{2} \ln \left(\frac{Q}{2 Q_{0}}\right)-g_{1} g_{3} b \ln \left(100 x_{A} x_{B}\right)\right\},
$$

where $g_{1}=0.11 \mathrm{GeV}^{2}, g_{2}=0.58 \mathrm{GeV}^{2}, g_{3}=-1.5 \mathrm{GeV}^{-1}$ and $Q_{0}=1.6 \mathrm{GeV}$. To consistently compare the distributions of the leptons in NLO and resummed calculations, we have used exactly the same PDF's, QCD and electroweak parameters, etc., for calculating the NLO results. F Furthermore, we have applied the kinematic cuts $p_{T}^{\ell}>25 \mathrm{GeV}, E_{T}>25 \mathrm{GeV}$, and $Q_{T}<20 \mathrm{GeV}$. These cuts are similar to those applied by the CDF group in the measurement of the asymmetry in the lepton rapidity distribution from $W$-boson decays. P]

The transverse momentum distributions of the charged lepton $p_{T}^{\ell}$ are shown in Fig. 1 for NLO and resummed calculations. We note that in these results a BreitWigner resonant width has been included, cf. Eq. (3). In the vicinity of $p_{T}^{\ell}=M_{W} / 2$ ("Jacobian peak") the NLO calculation is ill-defined because its amplitude blows up as $Q_{T} \rightarrow 0$. The effect of the initial state multiple soft-gluon emission on the distribution of $p_{T}^{\ell}$ is to widen and smoothen the "Jacobian peak" and therefore make it more challenging to accurately extract $M_{W}$ from the $p_{T}^{\ell}$ distribution.

Recently, the Fermilab CDF group measured the asymmetry $\mathcal{A}_{y^{\ell}}$ in the rapidity $y^{\ell}$ distribution of the charged lepton $(\ell)$ from the decay of $W^{+} \rightarrow \ell^{+} \nu_{\ell}\left(\right.$ or $W^{-} \rightarrow \ell^{-} \bar{\nu}_{\ell}$ ) [15] and proved this measurement to be particularly sensitive to the slope of the ratio of $u$ - to $d$-quark parton densities inside the proton [16, 17]. Define the asymmetry in the lepton rapidity distribution as

$$
\mathcal{A}_{y^{\ell}}=\frac{d \sigma / d y^{\ell}\left(y^{\ell}>0\right)-d \sigma / d y^{\ell}\left(y^{\ell}<0\right)}{d \sigma / d y^{\ell}\left(y^{\ell}>0\right)+d \sigma / d y^{\ell}\left(y^{\ell}<0\right)},
$$

\footnotetext{
7 These values were fit for CTEQ2M PDF, and in principle should be refit for CTEQ3M PDF.

8 Our NLO results agree with those in Ref. [14].

9 The requirement of $Q_{T}<20 \mathrm{GeV}$ in our calculation is approximately equivalent to cutting out the events in which the transverse momentum of the net hadronic activities reconstructed from the calorimeter cells within the pseudo-rapidity range of \pm 3.5 is larger than $20 \mathrm{GeV}$.
} 


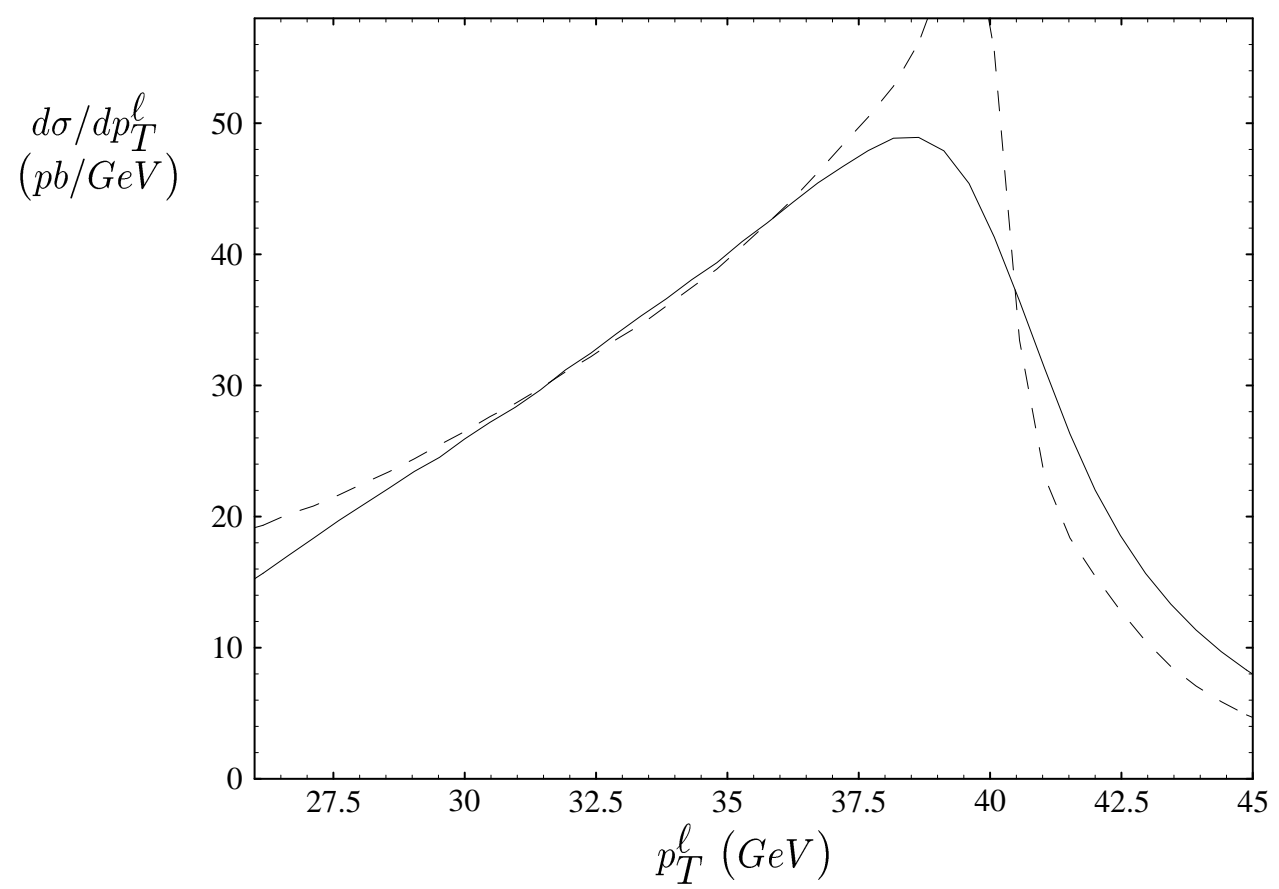

Figure 1: Transverse momentum distribution of the charged lepton $p_{T}^{\ell}$ for NLO (dashed) and resummed (solid) calculations. Resumming the initial state multiple soft-gluon emission has the typical effect of smoothening and widening the Jacobian peak (at $\left.p_{T}^{\ell}=M_{W} / 2\right)$. The NLO distribution is singular and ill-defined near $M_{W} / 2$.

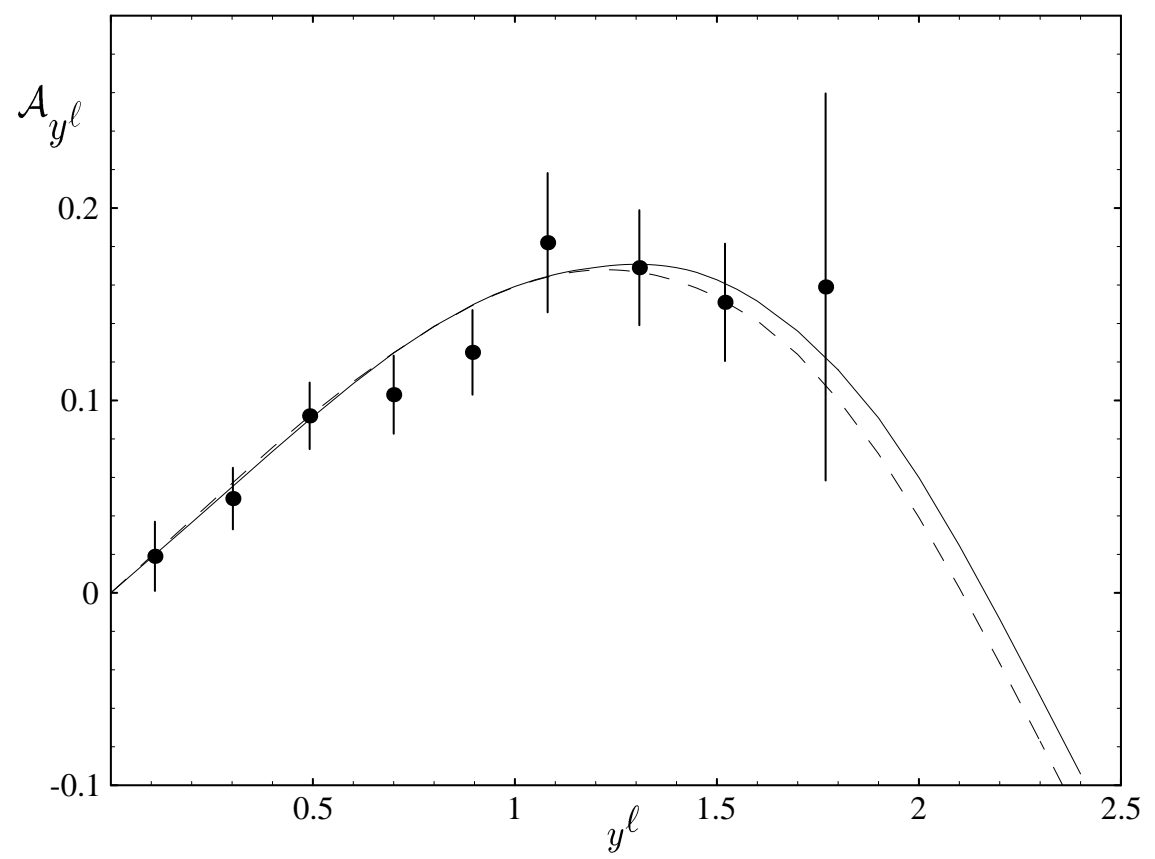

Figure 2: The asymmetry $\mathcal{A}_{y^{e}}$ in the lepton rapidity distribution as a function of $y^{\ell}$ for NLO (dashed) and resummed (solid) calculations. They differ the most in the large rapidity region $\left(\left|y_{\ell^{+}}\right|>1\right)$. The experimental data were obtained from Ref. [15] 


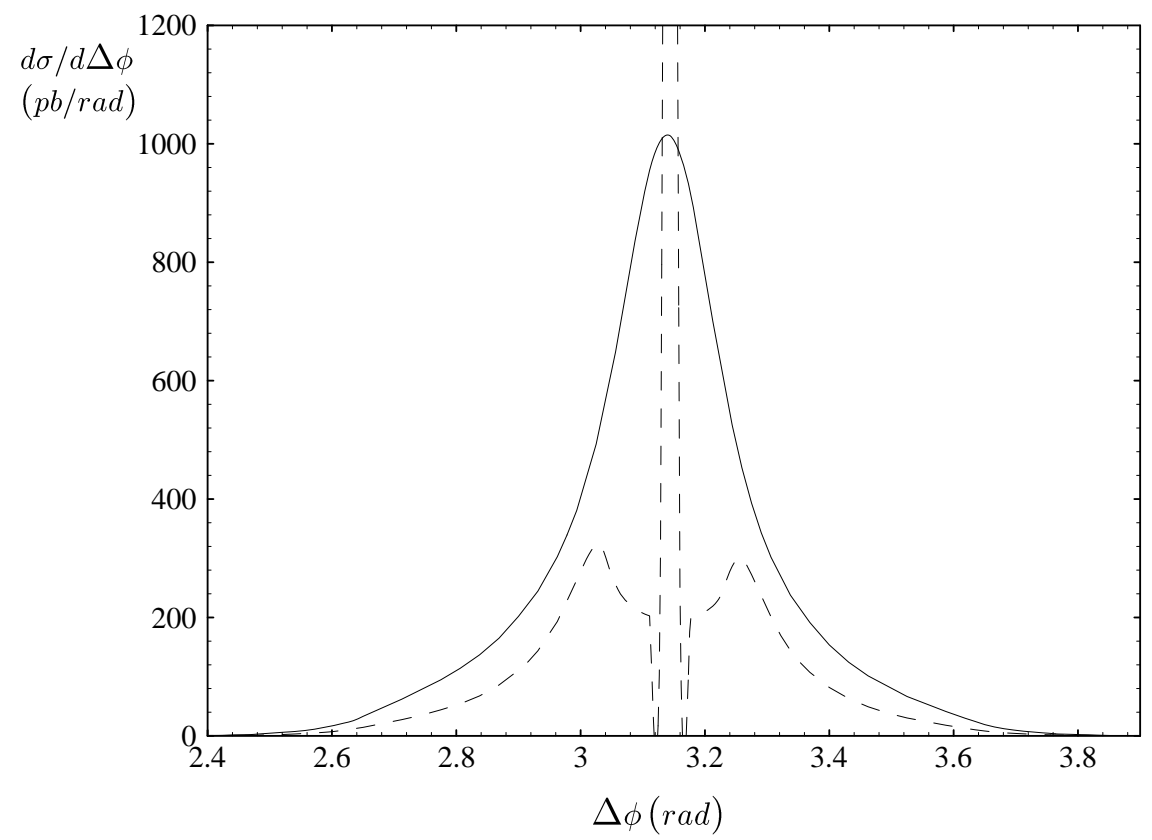

Figure 3: The distribution of the difference in the lepton azimuthal angles near the region $\Delta \phi \sim \pi$. The NLO (dashed) distribution is ill-defined at $\Delta \phi=\pi$ and is arbitrary around it. The resummed (solid) distribution gives the correct angular correlation of the lepton pair. The distribution has a similar peak for $\Delta \phi \sim-\pi$.

which is commonly known as the lepton charge asymmetry. In Fig. 2, we show $\mathcal{A}_{y^{\ell}}$ as a function of $y^{\ell}$ for NLO and resummed calculations. As indicated in the figure, they differ the most in the large rapidity region $\left(\left|y_{\ell^{+}}\right|>1\right)$. Recall that as $Q_{T} \rightarrow 0$ the NLO $Q_{T}$ distribution becomes singular, but the resummed result remains finite. The rapidity $y$ distribution of the $W$-boson in the NLO calculation is not singular, and we expect that after integrating out the complete phase space for $Q_{T}$ (that is, without imposing any kinematic cuts) the NLO and the resummed calculations should predict the same $y$ distributions. We have explicitly checked that this indeed is the case. However, in Fig. 2 some kinematic cuts (as described at the beginning of this section) have been applied to our calculations. In the rest (Collins-Soper) frame of the $W$-boson, the decay kinematics of the lepton $\ell$ is identical for both the NLO and the resummed calculations because the decay leptons do not involve strong interactions. Since the $W$-bosons have different kinematic distributions (e.g., $Q_{T}$ distributions) in these two calculations, the resulting lepton kinematic distributions 
(e.g., $y^{\ell}$ distributions) in the laboratory frame are different. The two $y^{\ell}$ distributions differ the most in the large $y^{\ell}$ region, where the typical $y$ is large, since the effects of soft-gluon emission become more important, close to the boundary of the phase space.

Another interesting observable to test the QCD theory beyond the fixed-order perturbative calculation is the measurement of the difference in the azimuthal angles of $l$ and $\bar{l}^{\prime}$ from the decay of $V$. In practice, this is better measured for $Z \rightarrow \ell^{+} \ell^{-}$. For the sake of argument, we show in Fig. 3 the difference $(\Delta \phi)$ in the azimuthal angles of $\ell^{+}$and $\nu_{\ell}$ measured in the laboratory frame for $W^{+} \rightarrow \ell^{+} \nu_{\ell}$ and calculated in NLO and resummed approaches. As clearly indicated, the NLO result is ill-defined in the vicinity of $\Delta \phi \sim \pm \pi$, where the multiple soft-gluon radiations have to be resummed to obtain physical predictions. Therefore, the transverse mass distributions of $\ell^{+}-\nu_{\ell}$ pair for NLO and resummed calculations are also different, $\square$ and in principle only the resummed results can sensibly predict the distributions of the leptons for a precise measurement of $M_{W}$.

In conclusion, we found that the distributions $\left(p_{T}^{\ell}, y^{\ell}\right.$ and $\left.\Delta \phi\right)$ of leptons are different in NLO and resummed calculations. For a better measurement of $M_{W}$ and $A_{y^{\ell}}$, the effects of the initial state multiple soft-gluon emission have to be considered in hadron collisions. The more detailed phenomenological studies will be presented elsewhere.

\section{Acknowledgments}

We thank E.L. Berger, R. Brock, G.A. Ladinsky, Wu-Ki Tung, and the CTEQ collaboration for many invaluable discussions. This work was supported in part by NSF under grant PHY-9309902 and by DOE under grant DE-FG02-92ER40730 and DE-FG02-87ER40731.

\footnotetext{
10 The transverse mass of $\ell^{+}-\nu_{\ell}$ pair is defined as $m_{T}^{\ell \nu}=\sqrt{2 p_{T}^{\ell} p_{T}^{\nu}(1-\cos \Delta \phi)}$.
} 


\section{Appendix}

Let us define the $q \bar{q}^{\prime} V$ and the $l \overline{l^{\prime}} V$ vertices, respectively, as

$$
i \gamma_{\mu}\left[g_{L}\left(1-\gamma_{5}\right)+g_{R}\left(1+\gamma_{5}\right)\right] \text { and } i \gamma_{\mu}\left[f_{L}\left(1-\gamma_{5}\right)+f_{R}\left(1+\gamma_{5}\right)\right] \text {. }
$$

For example, for $V=W^{+}, q=u, \bar{q}^{\prime}=\bar{d}, l=\nu_{e}$, and $\bar{l}^{\prime}=e^{+}$, the couplings $g_{L}^{2}=f_{L}^{2}=G_{F} M_{W}^{2} / \sqrt{2}$ and $g_{R}^{2}=f_{R}^{2}=0 .\left(G_{F}\right.$ is the Fermi constant.) In Eq. (11), for $N=1$,

$$
R_{a b}^{(1)}=\frac{16\left|V_{j k}\right|^{2}}{\pi Q^{2}}\left[\left(g_{L}^{2}+g_{R}^{2}\right)\left(f_{L}^{2}+f_{R}^{2}\right) R_{1}^{a b}+\left(g_{L}^{2}-g_{R}^{2}\right)\left(f_{L}^{2}-f_{R}^{2}\right) R_{2}^{a b}\right],
$$

where the coefficient functions $R_{i}^{a b}$ are given as follows:

$$
\begin{aligned}
& R_{1}^{j \bar{k}}=r^{j \bar{k}} \mathcal{L}_{0}+\frac{\mathcal{R}_{+}(t, u)}{s} \delta\left(s+t+u-Q^{2}\right)\left[\mathcal{A}_{0}+\mathcal{A}_{2}+\frac{Q}{Q_{T}} \mathcal{A}_{1}\right] \frac{Q^{2}}{M_{T}^{2}}, \\
& R_{2}^{j \bar{k}}=r^{j \bar{k}} \mathcal{A}_{3}+\frac{\mathcal{R}_{+}(t, u)}{s} \delta\left(s+t+u-Q^{2}\right) \\
& \times\left\{\frac{Q^{2}}{Q_{T}^{2}}\left(\frac{Q}{M_{T}}-1\right) \mathcal{A}_{3}-\frac{2 Q^{2}}{Q_{T} M_{T}} \frac{\mathcal{R}_{-}(t, u)}{\mathcal{R}_{+}(t, u)} \mathcal{A}_{4}\right\}, \\
& R_{1}^{g j}=r^{g j} \mathcal{L}_{0}-\frac{Q^{2} Q_{T}^{2}}{u M_{T}^{2}} \frac{\mathcal{R}_{+}(u, s)}{s} \delta\left(s+t+u-Q^{2}\right) \\
& \times\left\{\frac{\mathcal{R}_{+}(u,-s)}{\mathcal{R}_{+}(u, s)}\left[\mathcal{A}_{0}+\mathcal{A}_{2}\right]+\frac{Q}{Q_{T}} \frac{\left(Q^{2}-u\right)^{2}+\mathcal{R}_{-}(u, t)}{\mathcal{R}_{+}(u, s)} \mathcal{A}_{1}\right\}, \\
& R_{2}^{g j}=r^{g j} \mathcal{A}_{3}-\frac{Q_{T}^{2}}{u} \frac{\mathcal{R}_{+}(u, s)}{s} \delta\left(s+t+u-Q^{2}\right) \\
& \times\left\{\frac{Q^{2}}{Q_{T}^{2}}\left[\frac{Q}{M_{T}}\left(\frac{2 u\left(Q^{2}-s\right)}{\mathcal{R}_{+}(u, s)}-1\right)-1\right] \mathcal{A}_{3}\right. \\
& \left.-\frac{2 Q^{2}}{Q_{T} M_{T}}\left[\frac{2 s\left(Q^{2}-s\right)}{\mathcal{R}_{+}(u, s)}+1\right] \mathcal{A}_{4}\right\},
\end{aligned}
$$

with

$$
\begin{aligned}
r^{j \bar{k}}=\frac{Q^{2}}{Q_{T}^{2}}\left\{\frac{\mathcal{R}_{+}(t, u)}{s} \delta\left(s+t+u-Q^{2}\right)-2 \delta\left(1-z_{A}\right) \delta\left(1-z_{B}\right)\left[\ln \left(\frac{Q^{2}}{Q_{T}^{2}}\right)-\frac{3}{2}\right]\right. \\
\left.-\delta\left(1-z_{A}\right)\left(\frac{1+z_{B}^{2}}{1-z_{B}}\right)_{+}-\delta\left(1-z_{B}\right)\left(\frac{1+z_{A}^{2}}{1-z_{A}}\right)_{+}\right\}
\end{aligned}
$$

and

$$
r^{g j}=\frac{Q^{2}}{Q_{T}^{2}}\left\{-\frac{Q_{T}^{2}}{u} \frac{\mathcal{R}_{+}(u, s)}{s} \delta\left(s+t+u-Q^{2}\right)-\left[z_{A}^{2}+\left(1-z_{A}\right)^{2}\right] \delta\left(1-z_{B}\right)\right\},
$$


where $\mathcal{R}_{ \pm}(t, u)=\left(Q^{2}-t\right)^{2} \pm\left(Q^{2}-u\right)^{2}$. The angular dependence is described by the functions

$$
\begin{aligned}
& \mathcal{L}_{0}=1+\cos ^{2} \theta, \mathcal{A}_{0}=\frac{1}{2}\left(1-3 \cos ^{2} \theta\right), \mathcal{A}_{1}=\sin 2 \theta \cos \phi, \mathcal{A}_{2}=\frac{1}{2} \sin ^{2} \theta \cos 2 \phi \\
& \mathcal{A}_{3}=2 \cos \theta, \mathcal{A}_{4}=\sin \theta \cos \phi
\end{aligned}
$$

of which $\mathcal{A}_{3}$ and $\mathcal{A}_{4}$ are odd under parity operation.

\section{References}

[1] CDF Collaboration (F. Abe, et al.), Phys. Rev. Lett. 74 (1995) 2626

DØ Collaboration (S. Abachi, et al.), Phys. Rev. Lett. 74 (1995) 2632.

[2] Review of Particle Properties, Phys. Rev. D 50 (1994) 1173.

[3] Morris L. Swartz, SLAC preprint SLAC-PUB-6710, November 1994.

[4] J. Rosner, report EFI 94-38, August 1994,

[5] G. Altarelli, R.K. Ellis, M. Greco, G. Martinelli, Nucl. Phys. B 246 (1984) 12.

[6] J. Collins and D. Soper, Nucl. Phys. B 193 (1981) 381; Erratum B 213 (1983) 545; B 197 (1982) 446.

[7] J. Collins, D. Soper and G. Sterman, Nucl. Phys. B 250 (1985) 199.

[8] G. t'Hooft and M. Veltman, Nucl. Phys. B 44 (1972) 189;

P. Breitenlohner and D. Maison, Comm. Math. Phys. 52 (1977) 11.

[9] J.G. Körner, G. Schuler, G. Kramer, B. Lampe, Z. Phys. C 32 (1986) 181.

[10] J.G. Körner, E. Mirkes, G. Schuler, Internat. J. of Mod. Phys. A Vol. 4, No.7 (1989) 1781.

[11] J. Collins and D. Soper, Phys. Rev. D 16 (1977) 2219.

[12] C.S. Lam, Wu-Ki Tung, Phys. Rev. D 18 (1978) 2447. 
[13] G.A. Ladinsky and C.-P. Yuan, Phys. Rev. D 50 (1994) 4239 .

[14] W. Giele, E.Glover, D.A. Kosover, Nucl. Phys. B 403 (1993) 633.

[15] M. Dickson, Tests of Structure Functions using Leptons with CDF, in: HighEnergy Physics. Proceedings, International Europhysics Conference (Marseille, France, July 22-28, 1993), ed. J. Carr, M. Perrottet (Gif-sur-Yvette, France, 1994)

[16] E.L. Berger, F. Halzen, C.S. Kim, S. Willenbrock, Phys. Rev. D 40 (1989) 83; erratum, ibid. D 40 (1989) 3789.

[17] H.L. Lai, J. Botts, J. Huston, J.G. Morfin, J.F. Owens, J.W. Qiu, W.K. Tung, H. Weerts, MSU preprint MSU-HEP-41024, Oct 1994. 